\title{
Pancreas Neuroendocrine Tumor and Its Mimics: Review of Cross-Sectional Imaging Findings for Differential Diagnosis
}

\author{
Seung Soo Kim \\ Department of Radiology, Soonchunhyang University Cheonan Hospital, Soonchunhyang University College of Medicine, Cheonan, Korea
}

\begin{abstract}
The most common hypervascular neoplasm of the pancreas is neuroendocrine tumor (NET). Microcystic serous cystadenomas, certain metastases, and accessory spleens can also show hypervascularity and can mimic pancreatic NET. It is important to discriminate hypervascular pancreatic lesions because of different treatment option and prognosis. Although computed tomography (CT) is the most common imaging modality for initial identification of pancreatic tumor, $\mathrm{CT}$ alone cannot correctly interpret hypervascular pancreatic lesions. Therefore, when a hypervascular tumor in the pancreas is detected on $\mathrm{CT}$, magnetic resonance imaging should be considered. In this essay, I describe imaging features those are helpful for differential diagnosis of NET from other hypervascular lesions in pancreas.
\end{abstract}

Keywords: Pancreatic neoplasms; Neuroendocrine tumors; Multidetector computed tomography; Magnetic resonance imaging; Differential diagnosis

\section{INTRODUCTION}

Advances in diagnostic imaging and its increasing accessibility have led to an increase in the incidental detection of pancreatic tumors. In particular, computed tomography (CT) images can allow not only the detection but also the characterization of pancreatic lesions [1]. In the pancreatic phase, pancreas parenchyma shows peak enhancement and a considerable number of pancreatic tumors reveal lower attenuation than the pancreas parenchyma, making them easy to detect $[2,3]$. If a tumor shows higher attenuation than the pancreas parenchyma in the pancreatic phase, it can be considered to be a hypervascular lesion. The most common hypervascular tumor in the pancreas is a neuroendocrine tumor (NET) [4]. However, microcystic serous cystadenoma, certain metastases, and accessory spleen can also show hypervascularity in the pancreatic phase. The treatment options of NET and hypervascular metastases are surgical resection or chemoradiation therapy. On the other hand, microcystic serous cystadenoma and accessory spleen do not need further treatment, unless there is any symptom [5-7]. Therefore, it is important to correctly interpret them. The objectives of this article are to discuss pancreatic NET and its mimics and to illustrate imaging features those are helpful for differential diagnosis.

\section{NEUROENDOCRINE TUMOR}

Pancreatic NETs account for less than 5\% of pancreatic tumors [8]. A NET develops sporadically as a solitary lesion, although multiple NETs often develop in patients with a systemic disorder such as multiple endocrine neoplasia type 1, von Hippel-Lindau syndrome, neurofibromatosis type 1 , or tuberous sclerosis [4]. NETs usually present as an avidly enhancing mass and are characteristically best seen on arterial or pancreatic phase images (Figs. 1A, 2C). The enhancement of NETs persists in the venous phase (Figs. 1B, 2D) [9]. On magnetic resonance (MR) imaging, NETs generally appear hypointense and hyperintense on T1- and T2-weighted images, respectively (Fig. 2A, B). Because pancreatic NETs do not arise from the ductal epithelium, pancreatic ductal dilatation is unusual in patients with a NET (Fig. 2B) [10]. Although it is a challenge to discriminate between benign and malignant NETs, a malignant 

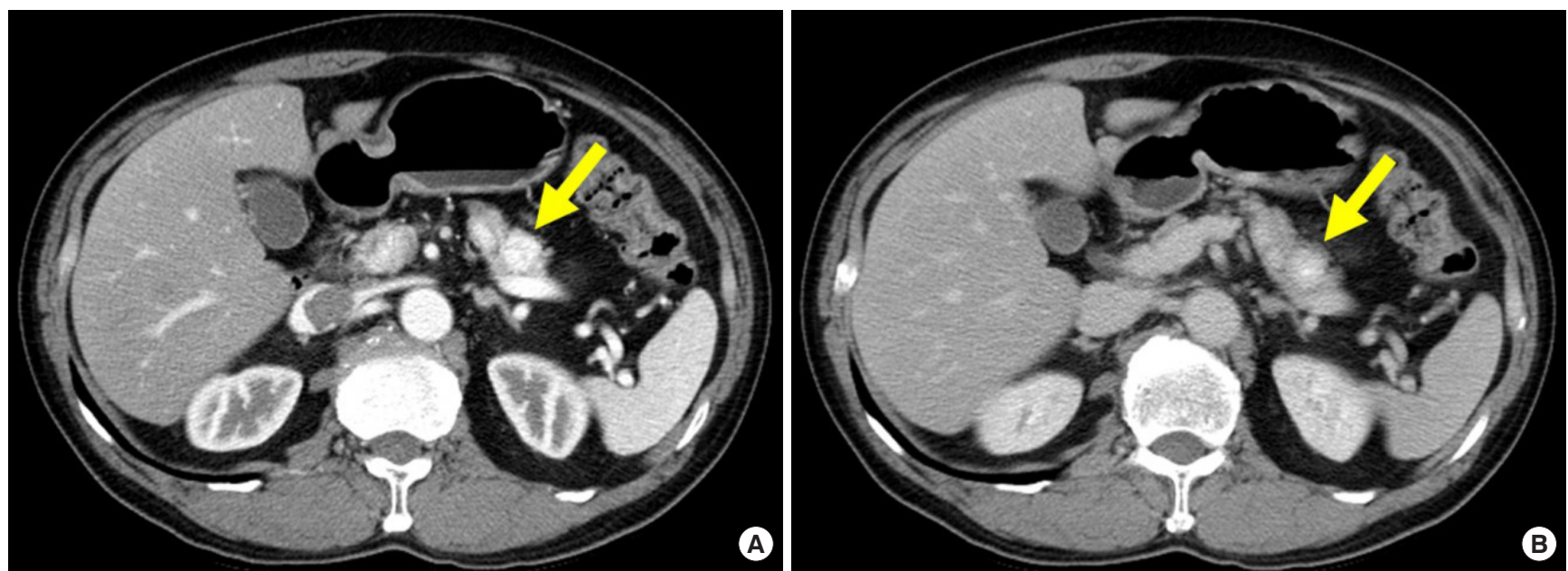

Fig. 1. A 62-year-old man with surgically diagnosed pancreatic insulinoma, who had symptoms of hypoglycemia. (A) Axial pancreatic phase CT image shows a 1.5-cm avidly enhancing mass (arrow) in the pancreatic body. (B) Axial portal venous phase CT image shows persistent enhancement of the mass (arrow). CT, computed tomography.
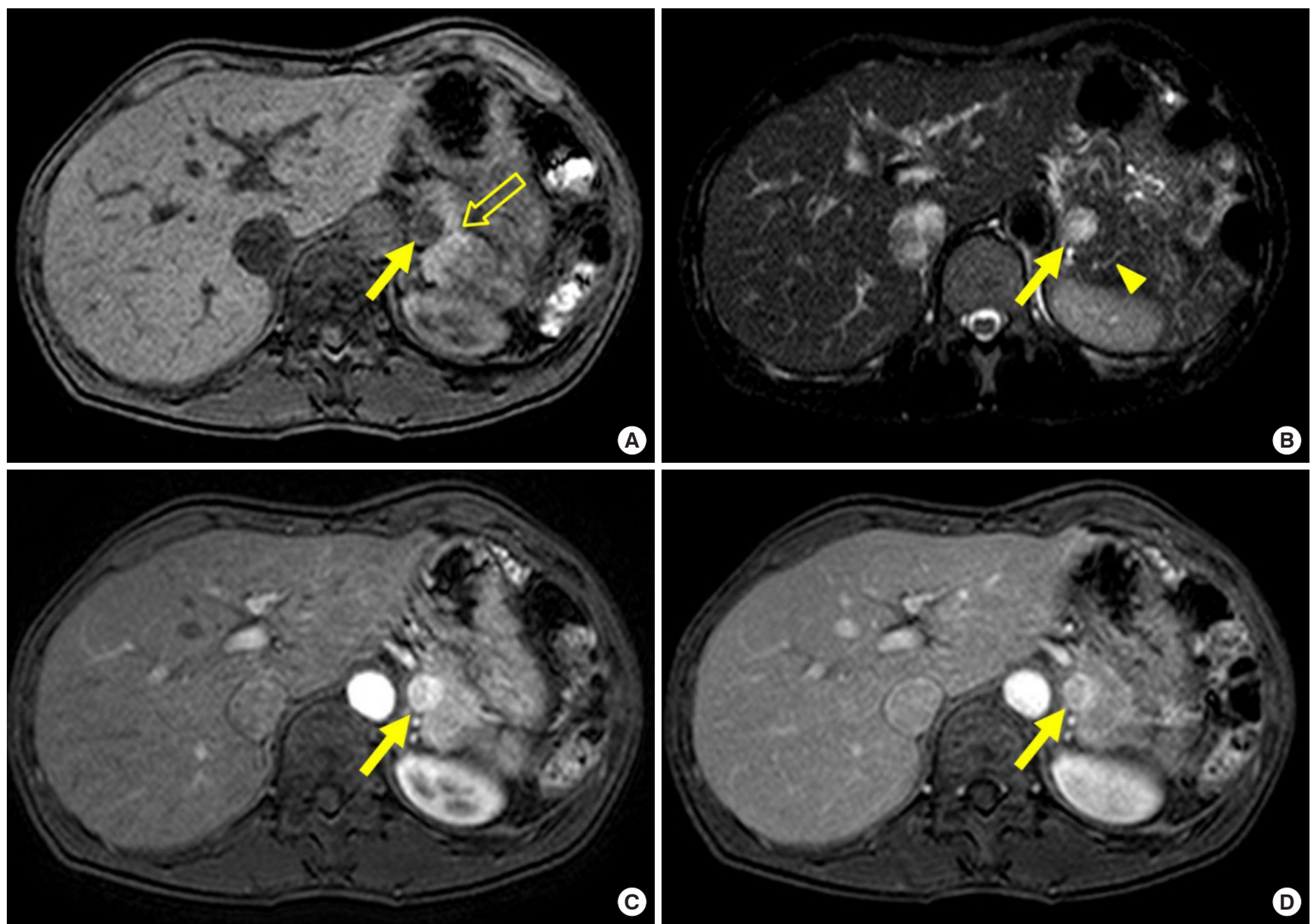

Fig. 2. A 48-year-old woman with pathologically confirmed pancreatic insulinoma. (A) Axial T1-weighted fat-suppressed MR image shows a 1.5-cm hypointense mass (arrow) in the pancreatic tail, which is well seen relative to the normal hyperintense pancreas (open arrow). (B) Axial T2-weighted fat-suppressed MR image shows higher signal intensity in the mass (arrow) relative to the pancreas. Note no dilatation of the pancreatic duct (arrowhead). (C) Axial arterial phase T1-weighted fat-suppressed MR image shows the mass (arrow) with marked enhancement. (D) Axial venous phase T1-weighted fat-suppressed MR image show persistent enhancement of the mass (arrow). MR, magnetic resonance. 
NET is suggested by features such as a tumor size larger than $3 \mathrm{~cm}$, complex cystic change, calcification, vascular invasion, pancreatic duct dilatation, and lymph node enlargement [11].

\section{MICROCYSTIC SEROUS CYSTADENOMA}

Serous cystadenomas account for $10 \%-16 \%$ of cystic lesions in the pancreas and are frequently detected in elderly woman [12]. Although a few serous cystadenocarcinomas have been reported, this cystic neoplasm is considered a benign lesion [12]. Serous cystadenomas are divided into polycystic (70\%), microcystic (20\%), and oligocystic $(<10 \%)$ types according to their morphology [13]. Among those morphologic types, the microcystic type histopathologically shows a sponge-like configuration composed of innumerous tiny cysts [14]. This lesion can mimic a hypervascular solid tumor such as a NET on CT images because it shows avid enhancement in the pancreatic phase (Fig. 3A) [15]. However, it demonstrates bright signal intensity on T2-weighted MR images, reflecting the cystic component, with no diffusion restriction (Fig. 3B, C) [5]. Therefore it is not difficult to differentiate microcystic serous cystadenoma from other solid tumors using MR imaging [5].

\section{PANCREATIC METASTASES FROM RENAL CELL CARCINOMA}

Metastases to the pancreas are not uncommon; a previous report demonstrated that $3 \%-12 \%$ of patients with malignancy at autopsy have them [16]. Renal cell carcinoma (RCC) is one of the common origins of pancreatic metastases, and a solitary pancreatic metastasis is common, especially in patients with the clear cell type of RCC [9]. Pancreatic metastases from RCC (pRCC) usually show arterial hyperenhancement and occur after several years (Fig. 4) [9]. Therefore, it is difficult to differentiate NETs from pRCC. Previous studies reported that pRCC showed arterial hyperenhancement followed by rapid washout and a recent study described that a relative washout value of more than $19 \%$ of tumor enhancement between the arterial and venous phases may be useful in discriminating pRCC from NETs (Fig. 4) [6,9]. In addition to enhancement pattern, MR imaging allows investigator to distinguish pRCC from microcystic serous cystadenoma (Fig. 4C).

\section{ACCESSORY SPLEEN}

Accessory spleen is a common anatomical variation that is frequently detected in daily practice [17]. These lesions are composed of red and white pulp, similar to the mother spleen, and show arterial hyperenhancement [7]. Most accessory spleens are located around the splenic hilum, but approximately $16 \%$ of them are detected in and around the pancreas tail, where they can mimic NETs (Fig. 5A) [18]. However, accessory spleens reveal signal intensity and enhancement patterns that are similar to those of the mother spleen in all MR imaging sequences (Fig. 5B-F) [7]. They can be diagnosed by finding radio-uptake on Tc-99m heat-damaged red blood cell scintigraphy without pathologic confirmation (Fig. 5G) [7]. Radiologists have to consider the possibility of accessory spleen when a hypervascular lesion is detected in the pancreas.

\section{CONCLUSION}

The most common hypervascular tumors in pancreas are
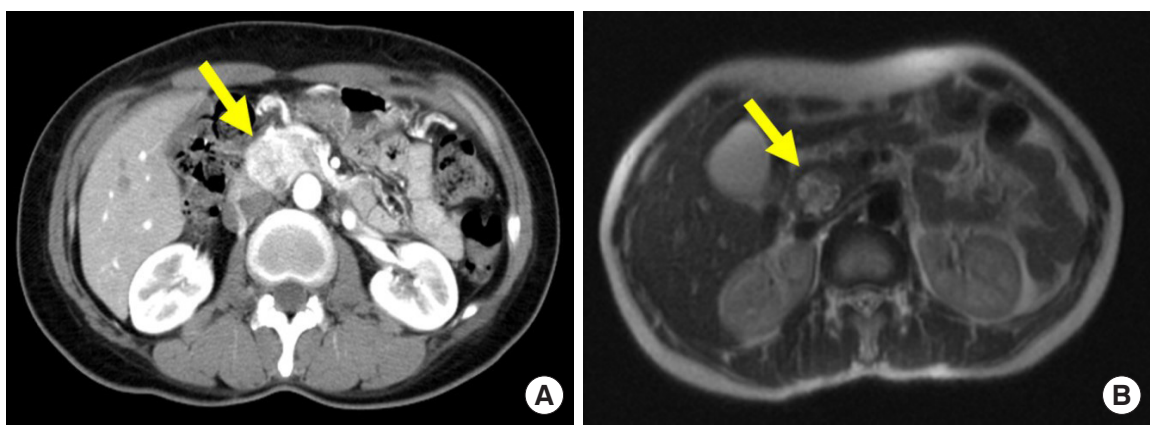

Fig. 3. A 49-year-old woman with surgically proven microcystic serous cystadenoma. (A) Axial pancreatic phase computed tomography image shows 3-cm avidly enhancing mass (arrow) in the pancreatic head. (B, C) Axial T2-weighted magnetic resonance imaging and magnetic resonance cholangiopancreatography images show a multiseptated cystic lesion (arrow) in the pancreatic tail.

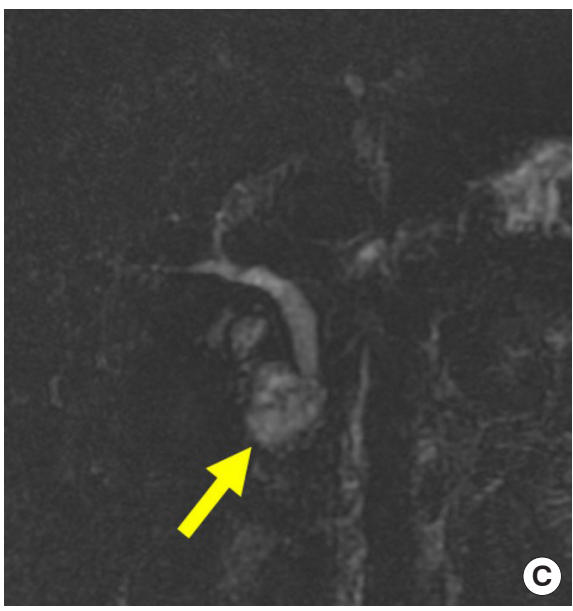



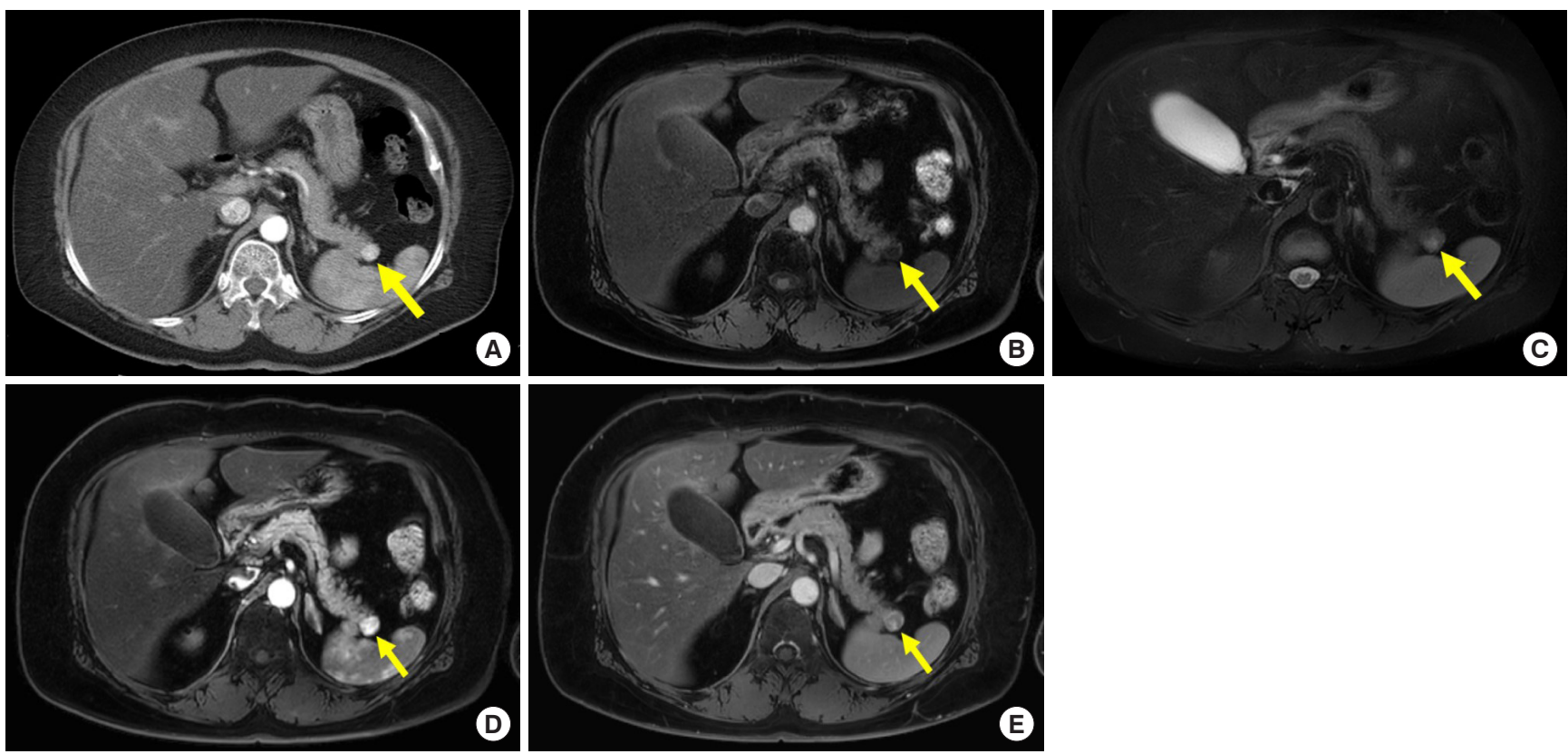

Fig. 4. A 54-year-old woman with surgically diagnosed pancreatic metastases from renal cell carcinoma who had a history of nephrectomy 20 years ago. (A) Axial arterial phases computed tomography images show 1.3-cm hypervascular mass (arrow) in pancreatic tail. (B) Axial T1-weighted fat-suppressed MR image shows hypointense mass (arrow) with well-defined margin in pancreatic tail. (C) On axial T2-weighted fat-suppressed MR image, the mass (arrow) is isointense with some slightly hyperintense foci compared with pancreas parenchyma, therefore that is not microcystic serous cystadenoma. (D, E) On axial arterial and venous phase contrast-enhanced MR images, the mass (arrow) shows arterial hyperenhancement with followed washout. The mean relative washout value between arterial and venous phase of the pancreatic masses was $46.1 \%$. MR, magnetic resonance.
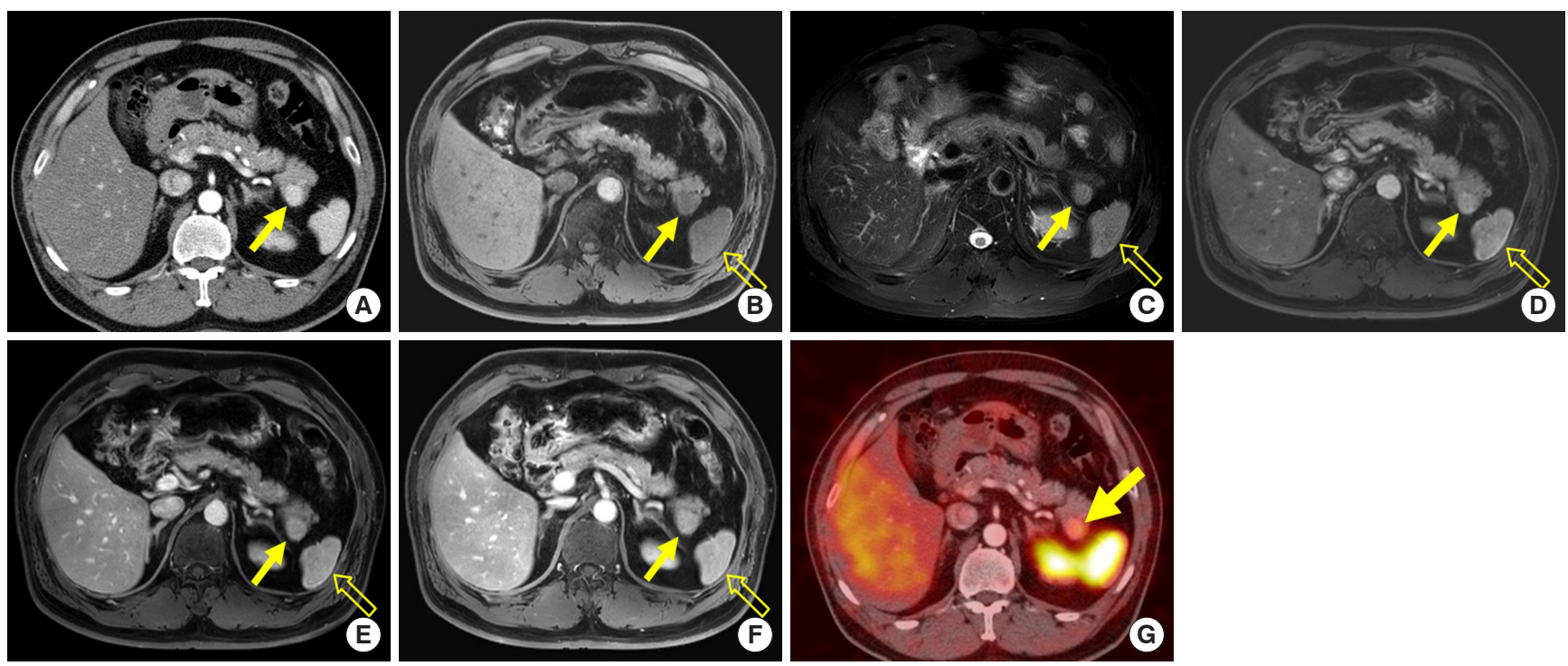

Fig. 5. A 52-year-old man with an accessory spleen in the pancreatic tail. (A) Axial arterial phase computed tomography image shows a 2.3-cm avidly enhancing mass (arrow) in the pancreatic tail. (B, C) On axial T1- and T2-weighted fat-suppressed MR images, the mass (arrow) reveals signal intensity similar to that of the spleen (open arrow). (D-F) On axial dynamic contrast-enhanced MR images, the enhancement pattern of the mass (arrow) was similar to that of the adjacent spleen (open arrow). (G) Damaged red blood cell scintigraphy shows intense uptake in the mass (arrow). MR, magnetic resonance.

NETs, and microcystic serous cystadenomas, hypervascular metastases, and accessory spleen can mimic pancreatic NETs. Microcystic serous cystadenoma and accessory spleen can be easily dif- ferentiated from NET or pancreatic metastases using MR imaging. Therefore, MR imaging should be considered when pancreas hypervascular tumors are detected on CT. Tumor enhancement 
pattern is helpful for differentiating pRCC from NETs. Differentiation of pancreatic NET and its mimics is vital for managing patients in daily practice and radiologists must be aware of imaging features of them.

\section{ACKNOWLEDGMENTS}

The author of this manuscript declares no relationships with any companies, whose products or services may be related to the subject matter of the article. No study subjects or cohorts have been previously reported in others. The authors declare no conflict of interest. Statement of informed consent was not applicable since the manuscript does not contain any patient data.

\section{REFERENCES}

1. Lu DS, Vedantham S, Krasny RM, Kadell B, Berger WL, Reber HA. Twophase helical CT for pancreatic tumors: pancreatic versus hepatic phase enhancement of tumor, pancreas, and vascular structures. Radiology 1996;199:697-701.

2. Fletcher JG, Wiersema MJ, Farrell MA, Fidler JL, Burgart LJ, Koyama T, et al. Pancreatic malignancy: value of arterial, pancreatic, and hepatic phase imaging with multi-detector row CT. Radiology 2003;229:81-90.

3. Kondo H, Kanematsu M, Goshima S, Miyoshi T, Shiratori Y, Onozuka M, et al. MDCT of the pancreas: optimizing scanning delay with a bolustracking technique for pancreatic, peripancreatic vascular, and hepatic contrast enhancement. AJR Am J Roentgenol 2007;188:751-6.

4. Lewis RB, Lattin GE Jr, Paal E. Pancreatic endocrine tumors: radiologicclinicopathologic correlation. Radiographics 2010;30:1445-64.

5. Park HS, Kim SY, Hong SM, Park SH, Lee SS, Byun JH, et al. Hypervascular solid-appearing serous cystic neoplasms of the pancreas: differential diagnosis with neuroendocrine tumours. Eur Radiol 2016;26:134858.

6. Ahmed S, Johnson PT, Hruban R, Fishman EK. Metastatic disease to the pancreas: pathologic spectrum and CT patterns. Abdom Imaging 2013; 38:144-53.

7. Kim SH, Lee JM, Han JK, Lee JY, Kim KW, Cho KC, et al. Intrapancreatic accessory spleen: findings on MR Imaging, CT, US and scintigraphy, and the pathologic analysis. Korean J Radiol 2008;9:162-74.

8. Halfdanarson TR, Rabe KG, Rubin J, Petersen GM. Pancreatic neuroendocrine tumors (PNETs): incidence, prognosis and recent trend toward improved survival. Ann Oncol 2008;19:1727-33.

9. Kang TW, Kim SH, Lee J, Kim AY, Jang KM, Choi D, et al. Differentiation between pancreatic metastases from renal cell carcinoma and hypervascular neuroendocrine tumour: use of relative percentage washout value and its clinical implication. Eur J Radiol 2015;84:2089-96.

10. Rha SE, Jung SE, Lee KH, Ku YM, Byun JY, Lee JM. CT and MR imaging findings of endocrine tumor of the pancreas according to WHO classification. Eur J Radiol 2007;62:371-7.

11. Gallotti A, Johnston RP, Bonaffini PA, Ingkakul T, Deshpande V, Fernandez-del Castillo C, et al. Incidental neuroendocrine tumors of the pancreas: MDCT findings and features of malignancy. AJR Am J Roentgenol 2013;200:355-62.

12. Jais B, Rebours V, Malleo G, Salvia R, Fontana M, Maggino L, et al. Serous cystic neoplasm of the pancreas: a multinational study of $2622 \mathrm{pa}-$ tients under the auspices of the International Association of Pancreatology and European Pancreatic Club (European Study Group on Cystic Tumors of the Pancreas). Gut 2016;65:305-12.

13. Sahani DV, Kadavigere R, Saokar A, Fernandez-del Castillo C, Brugge WR, Hahn PF. Cystic pancreatic lesions: a simple imaging-based classification system for guiding management. Radiographics 2005;25:1471-84.

14. Compagno J, Oertel JE. Microcystic adenomas of the pancreas (glycogen-rich cystadenomas): a clinicopathologic study of 34 cases. Am J Clin Pathol 1978;69:289-98.

15. Khan A, Khosa F, Eisenberg RL. Cystic lesions of the pancreas. AJR Am J Roentgenol 2011;196:W668-77.

16. Scatarige JC, Horton KM, Sheth S, Fishman EK. Pancreatic parenchymal metastases: observations on helical CT. AJR Am J Roentgenol 2001;176: 695-9.

17. Freeman JL, Jafri SZ, Roberts JL, Mezwa DG, Shirkhoda A. CT of congenital and acquired abnormalities of the spleen. Radiographics 1993;13: 597-610.

18. Halpert B, Gyorkey F. Lesions observed in accessory spleens of 311 patients. Am J Clin Pathol 1959;32:165-8. 